\title{
QCD constituent counting rules for neutral vector mesons
}

\author{
Stanley J. Brodsky, ${ }^{1}$ Richard F. Lebed, ${ }^{2}$ and Valery E. Lyubovitskij ${ }^{3,4,5,6}$ \\ ${ }^{1}$ SLAC National Accelerator Laboratory, Stanford University, Stanford, California 94309, USA \\ ${ }^{2}$ Department of Physics, Arizona State University, Tempe, Arizona 85287-1504, USA \\ ${ }^{3}$ Institut für Theoretische Physik, Universität Tübingen, Kepler Center for Astro and Particle Physics, \\ Auf der Morgenstelle 14, D-72076 Tübingen, Germany \\ ${ }^{4}$ Departamento de Física y Centro Científico Tecnológico de Valparaíso-CCTVal, \\ Universidad Técnica Federico Santa María, Casilla 110-V Valparaíso, Chile \\ ${ }^{5}$ Department of Physics, Tomsk State University, 634050 Tomsk, Russia \\ ${ }^{6}$ Laboratory of Particle Physics, Tomsk Polytechnic University, 634050 Tomsk, Russia
}

(Received 27 December 2017; published 8 February 2018)

\begin{abstract}
QCD constituent counting rules define the scaling behavior of exclusive hadronic scattering and electromagnetic scattering amplitudes at high momentum transfer in terms of the total number of fundamental constituents in the initial and final states participating in the hard subprocess. The scaling laws reflect the twist of the leading Fock state for each hadron and hence the leading operator that creates the composite state from the vacuum. Thus, the constituent counting scaling laws can be used to identify the twist of exotic hadronic candidates such as tetraquarks and pentaquarks. Effective field theories must consistently implement the scaling rules in order to be consistent with the fundamental theory. Here, we examine how one can apply constituent counting rules for the exclusive production of one or two neutral vector mesons $V^{0}$ in $e^{+} e^{-}$annihilation, processes in which the $V^{0}$ can couple via intermediate photons. In the case of a (narrow) real $V^{0}$, the photon virtuality is fixed to a precise value $s_{1}=m_{V^{0}}^{2}$, thus treating the $V^{0}$ as a single fundamental particle. Each real $V^{0}$ thus contributes to the constituent counting rules with $N_{V_{0}}=1$. In effect, the leading operator underlying the $V^{0}$ has twist 1 . Thus, in the specific physical case of single or double on-shell $V^{0}$ production via intermediate photons, the predicted scaling from counting rules coincides with vector-meson dominance (VMD), an effective theory that treats $V^{0}$ as an elementary field. However, the VMD prediction fails in the general case where the $V^{0}$ is not coupled through an elementary photon field, and then the leading-twist interpolating operator has twist $N_{V_{0}}=2$. Analogous effects appear in $p p$ scattering processes.
\end{abstract}

DOI: 10.1103/PhysRevD.97.034009

\section{INTRODUCTION}

One of the distinctive consequences of the underlying conformal features of gauge theories such as QCD is counting rules for hard exclusive processes. In such processes, one can factorize the physical scattering amplitude as the convolution of a hard-scattering quark and gluon amplitude $T_{H}$ with the product of hadronic distribution amplitudes $\phi_{H}(x, Q)$. The resulting scaling for the differential cross section at large momentum transfer reads [1-3] $d \sigma / d t \sim 1 / S^{N-2}$, where $S$ is a generic hard scale, and $N=N_{i}+N_{f}$ is the total number of fundamental constituents participating in the hard subprocess. The number of

Published by the American Physical Society under the terms of the Creative Commons Attribution 4.0 International license. Further distribution of this work must maintain attribution to the author(s) and the published article's title, journal citation, and DOI. Funded by SCOAP ${ }^{3}$. constituents of each hadron entering the scattering amplitude coincides with the number of particles in its leading Fock state and thus with the twist of the leading operator that creates the composite state from the vacuum. For example, the scaling prediction for exclusive cross sections such as fixed-angle hadron-hadron scattering is [1-3]

$$
\frac{d \sigma}{d t}(A+B \rightarrow C+D) \propto \frac{F\left(\theta_{\mathrm{CM}}\right)}{S^{N-2}}
$$

where $N=N_{A}+N_{B}+N_{C}+N_{D}$ is the total twist or number of elementary constituents. When dealing with hadrons, one must take into account their quark content and use $N_{M}=2$ and $N_{B}=3$ for each meson and baryon, respectively. One also predicts logarithmic corrections from the behavior of the running couplings entering $T_{H}$ and the Efremov-Radyushkin-Brodsky-Lepage (ERBL) evolution of the distribution amplitude. 


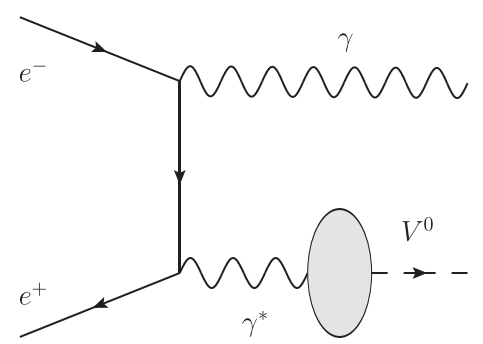

FIG. 1. Diagram for exclusive production of a vector meson $V^{0}$ in $e^{+} e^{-} \rightarrow \gamma V^{0}$, the corresponding $u$-channel diagram being implied.

The constituent counting rules are completely rigorous when they are applied properly. The leading-twist contribution to the power-law falloff of a cross section for any exclusive or semi-inclusive process depends upon the twist of the operators that couple the hadron to the hard subprocess. The twist $\tau$ of a hadron that couples to a hard-scattering subprocess is computed from the number $N$ of its fundamental constituent fields interacting in the hard-scattering subprocess (called active in Ref. [4]), plus $L$, the relative orbital angular momenta in the contributing hadronic Fock state. In contrast, the cross section for hadrons produced through a soft intermediate state- such as a neutral vector meson $V^{0}$ produced via its direct coupling to a photon of finite virtuality, or a hadron produced from jet fragmentation-does not have increased power-law falloff.

Note also that effective field theories developed to describe hard hadronic processes must consistently implement the counting rules in order to be consistent with the underlying fundamental theory of QCD. AdS/QCD, which allows the calculation of hadronic amplitudes using lightfront holography [5], is a good example of such an effective theory.

In Refs. [6,7], the present authors studied the application of the constituent counting rules for the production of tetraquarks, pentaquarks (as first suggested for the $\Lambda(1405)$ in Refs. [8,9]), and $V^{0}$ in the exclusive reactions of electroproduction and $p \bar{p}$ and $e^{-} e^{+}$annihilation. The purpose of the present paper is to further clarify our point regarding single and double on-shell $V^{0}$ production in $e^{+} e^{-}$ annihilation (see Figs. 1 and 2), where each $V^{0}$ couples to the hard subprocess via a virtual photon. In effect, the leading operator underlying the $V^{0}$ has twist $N_{V^{0}}=1$, a point not fully appreciated in Ref. [7]. In fact, the possibility that some of the constituents in a given process counted in the scaling rule might not be hard is the essence of the critique of [6] given in Ref. [10]. Thus, in the specific physical case of single or double $V^{0}$ production via intermediate photons, the predicted scaling from counting rules coincides with vector-meson dominance (VMD) [11], an effective theory that treats the $V^{0}$ 's as elementary fields. A modified form of the constituent counting rules therefore holds, and QCD can be approximated at these exceptional

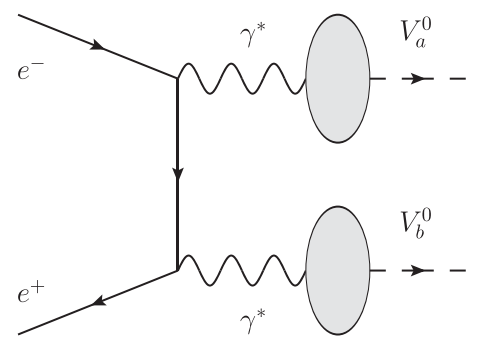

FIG. 2. Diagram for exclusive production of vector mesons $V_{a}^{0}$ and $V_{b}^{0}$ in $e^{+} e^{-} \rightarrow V_{a}^{0} V_{b}^{0}$, the corresponding $u$-channel diagram being implied.

kinematic points by an effective field theory, the VMD model, which treats $V^{0}$ as an elementary field. However, as we shall show, VMD, in general, is not consistent with QCD and the constituent counting rules. The VMD prediction fails in the general case in which the $V^{0}$ is not coupled to the hard subprocess via an elementary photon field; in that case, the leading-twist interpolating operator has twist $N_{V_{0}}=2$.

This paper is organized as follows: Section II provides general comments about the $e^{+} e^{-} \rightarrow \gamma^{*} \gamma$ and $\gamma^{*} \gamma^{*}$ processes, both leptonic and hadronic. In Sec. III we examine the process $e^{+} e^{-} \rightarrow \gamma \mu^{+} \mu^{-}$, for which the relevant cross sections have been explicitly computed and for which the high-momentum scaling behavior is explicit, and infer the corresponding behavior for $V^{0}$ production. Section IV shows how the original constituent counting rules persist in inclusive $e^{+} e^{-}$processes involving vector (or scalar or tensor) meson production. In Sec. V we consider applications of these ideas in $p p$ scattering processes, and in Sec. VI we conclude.

\section{VECTOR-MESON PRODUCTION VIA INTERMEDIATE PHOTONS}

The most straightforward scaling predicted by the counting rules is valid in most physical applications, e.g., in the pair production of mesons, baryons, or tetraquarks in $e^{+} e^{-}$annihilation [6], and it occurs whenever all constituents participate in the hard process, in which cases the scale $S$ is just Mandelstam $s$, the square of the total center-of-momentum (c.m.) energy. If any of the particles undergo hard scatterings that constrain them to lie in the forward (beam) c.m. direction, the corresponding factors of $S$ become Mandelstam $|t|$ [7].

However, specific physical cases exist, e.g., single or double vector-boson $V^{0}$ production processes $e^{+} e^{-} \rightarrow \gamma V^{0}$ and $e^{+} e^{-} \rightarrow V_{a}^{0} V_{b}^{0}$, in which each $V^{0}$ couples solely to an intermediate elementary photon field, $\gamma \rightarrow V^{0}$, or a weak gauge boson, $Z^{0} \rightarrow V^{0}, W^{ \pm} \rightarrow V^{ \pm}$. In such cases, the scale associated with the photon virtuality is fixed to a precise value $s_{1}=m_{V^{0}}^{2}$, where $m_{V^{0}}$ is the vector-meson mass. Therefore, one can treat the $V^{0}$ (with respect to the counting 
rules) as a single fundamental particle, and QCD reduces to the limit of the VMD model. In this specific case, the $V^{0}$ is approximated by an elementary field with $N_{V^{0}}=1$ elementary constituents. Then, one has $N-2=2$ for both processes $e^{+} e^{-} \rightarrow \gamma V^{0}$ and $e^{+} e^{-} \rightarrow V_{a}^{0} V_{b}^{0}$, which gives the differential cross-section scaling $d \sigma / d t \propto 1 / s^{2}$, where $s$ is the total c.m. energy of the lepton pair, or $1 / s|t|$ for forward scattering. This result follows from setting $s_{1}=$ $m_{V^{0}}^{2}$ in the $\gamma \rightarrow V^{0}$ transition form factor $G_{V}\left(s_{1}\right)$ (calculated, e.g., using the soft-wall AdS/QCD approach) in Ref. [7], rather than introducing an $O(|t|)$ scale in $G_{V}$ as advocated in that work. Independently, this scaling result can be shown explicitly by considering the related process $e^{+} e^{-} \rightarrow \gamma \mu^{+} \mu^{-}$at high energy but small invariant mass for the $\mu^{+} \mu^{-}$pair (Sec. III), an exercise that is instructive in explicitly indicating where the various momentum scales appear. Let us stress again that the scaling of the differential cross section $d \sigma / d t \propto 1 / s^{2}$ in the particular processes of single or double vector-boson $V^{0}$ production does not violate the constituent counting rules because the exclusive $\gamma \rightarrow V^{0}$ transition necessarily implies soft QCD, leading one to approximate the $V^{0}$ (with respect to hard scales) as an elementary field. In other words, the presence of soft QCD vertices in hard processes leads to a decrease of the scaling power in the corresponding differential cross section by identifying each softly produced hadron composed of $N_{a}$ constituents with an elementary field: $N_{a} \rightarrow 1$.

Note that the production of a $V^{0}$ via a photon can be a subleading contribution to the matrix element of a hard process. An example of such a process is $V^{0}$ production in the reaction $e^{+} e^{-} \rightarrow V^{0} P^{0}$, where $P^{0}$ is a neutral pseudoscalar meson (e.g., $\pi^{0}, \eta, \eta^{\prime}$ ). In Ref. [10], VMD was the mechanism proposed for the $\gamma \rightarrow V^{0}$ transition in such processes. It is clear that this subprocess is $O\left(\alpha_{\mathrm{em}}\right)$ suppressed in comparison with the leading QCD diagram discussed in Ref. [12] for direct production of a $V^{0} P^{0}$ pair by a hard photon, $\gamma^{*} \rightarrow V^{0} P^{0}$. As was shown in Ref. [12], the matrix element for $e^{+} e^{-} \rightarrow V^{0} P^{0}$ contains a helicityflip transition form factor $F_{\gamma^{*} V^{0} P^{0}}(s)$, which encodes violation of hadron helicity selection rules and scales as $1 / s^{2}$ at large $s$. As a result, the corresponding cross section scales as $d \sigma / d t \propto 1 / s^{5}$; i.e., it has an additional $1 / s$ falloff compared to helicity-favored modes of two-meson production $\left(\pi^{+} \pi^{-}, K^{+} K^{-}\right.$, etc.). The mechanism for the $e^{+} e^{-} \rightarrow$ $V^{0} P^{0}$ reaction considered in Ref. [10] gives $d \sigma / d t \propto 1 / s^{3}$, but, as stressed above, it is suppressed by a power of $\alpha_{\mathrm{em}}$ in comparison with the leading QCD diagram.

\section{LESSONS FROM THE PROCESS $e^{+} e^{-} \rightarrow \gamma \mu^{+} \mu^{-}$}

In order to verify or falsify the claim from Ref. [7] that the $\gamma^{*} V^{0}$ transition form factor $G_{V}\left(q^{2}\right)$ scales as $1 / \sqrt{|t|}$ for forward scattering in $e^{+} e^{-} \rightarrow \gamma V^{0}$, one may study the related process $e^{+} e^{-} \rightarrow \gamma \mu^{+} \mu^{-}$, which has been considered

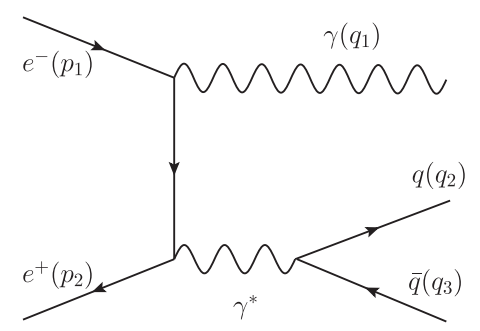

FIG. 3. Diagram contributing to $e^{+} e^{-} \rightarrow \gamma \gamma^{*} \rightarrow \gamma q \bar{q}$, the corresponding $u$-channel diagram being implied.

for decades $[13,14]$ as a background to $e^{+} e^{-} \rightarrow \mu^{+} \mu^{-}$, and more recently [15] in the initial-state radiation process, in which the real photon is hard but the $\mu^{+} \mu^{-}$pair is soft. Indeed, the result of Ref. [15] was used to estimate the yield of true muonium $\left(\mu^{+} \mu^{-}\right)$atoms in the process $e^{+} e^{-} \rightarrow$ $\gamma+\left(\mu^{+} \mu^{-}\right)$[16]. The process assumes the same topology as Fig. 3, with $q \bar{q}$ replaced by $\mu^{-} \mu^{+}$.

To serve as an orientation, we exhibit the textbook result [17] of the Born-level cross section for the pair-annihilation process $e^{+} e^{-} \rightarrow \gamma \gamma$. One finds, neglecting masses, and in the forward direction $\left(m_{e}^{2} \ll|t| \ll s\right)$,

$$
\frac{d \sigma}{d t} \rightarrow \frac{2 \pi \alpha^{2}}{s^{2}|t|} \frac{s^{2}+2 s t+2 t^{2}}{s+t} \rightarrow \frac{2 \pi \alpha^{2}}{s|t|}
$$

in agreement with the prediction of Eq. (1) with $N=4$ and one forward (fermion) propagator.

The full Born-level cross section for $e^{+} e^{-} \rightarrow \gamma+\left(\mu^{+} \mu^{-}\right)$, as can be seen in Eq. (14) of Ref. [14], possesses a secondorder pole $1 /(1 \pm z)^{2}$ in $z \equiv \cos \theta_{+}$, where $\theta_{+}$is the $\mu^{+} e^{-}$ angle in the $e^{+} e^{-}$c.m. frame. The corresponding differential cross section $d \sigma / d t$ therefore has separate terms scaling as $1 /|t|^{2}$ and as $1 /|u|^{2}$. They clearly arise through the near-collinear kinematics in which $\mu^{+}, \mu^{-}$, and $\gamma$ all lie close to the beam axis but have large relative momenta; in that case, both the fermion and photon propagators in Fig. 3 contribute the large momentum-transfer factors.

But now restrict to the kinematics of Ref. [15], in which the momentum transfer $s_{1}$ of the $\mu^{+} \mu^{-}$pair is small; in the exclusive $q \bar{q}$ case, $s_{1}=m_{V^{0}}^{2}$ (labeled $q^{2}$ in Ref. [7]). The $e^{+} e^{-} \rightarrow \gamma+\left(\mu^{+} \mu^{-}\right)$forward differential cross section then reads

$$
\frac{d \sigma}{d t}=\frac{\alpha^{3}}{s|t| s_{1}}\left(2 \delta+1-2 x_{-} x_{+}\right) d x_{-} d s_{1},
$$

where $\delta \equiv m_{\mu}^{2} / s_{1}, x_{ \pm} \equiv E_{\mu^{ \pm}} /(\sqrt{s} / 2)$ are the fractional $\mu^{ \pm}$ energies, and $m_{e} \rightarrow 0$. The question then becomes how much the remaining integrals, those over $d x_{-}$and $d s_{1}$, influence the full high-momentum scaling of $d \sigma / d t$. One easily finds that

$$
s_{1} \rightarrow 2 s \sin ^{2} \frac{\epsilon}{2}, \quad t \rightarrow-2 \sin ^{2} \frac{\theta}{2},
$$


where $\epsilon$ is the $\mu^{+} \mu^{-}$angle and $\theta$ is the $e^{-} \gamma$ angle in the c.m. frame. We are therefore interested in the hierarchy $s_{1} \ll$ $|t| \ll s$ or $\epsilon \ll \theta \ll 1$. Immediately one sees that small $s_{1}$ requires a small c.m. angle $\epsilon$ between the $\mu^{+} \mu^{-}$pair; however, at this stage no similar restriction requires the $\mu^{+}$ and $\mu^{-}$to share their total energy $\sqrt{s} / 2$ equally, so $x_{-}=$ $1-x_{+}$can assume any value $\in[0,1]$.

We now turn to the exclusive hadronic case $\left(\mu^{-} \mu^{+} \rightarrow q \bar{q}\right)$, in which Eq. (3) is modified via multiplication by a color factor 3 and the $\gamma^{*} V^{0}$ transition form factor $\left|G_{V}\right|^{2}$. Here, one may naively think that the constraint of forming a bound state - that the momenta of the initial $q \bar{q}$ pair differ by no more than $O\left(\sqrt{s_{1}}\right)=O\left(\Lambda_{\mathrm{QCD}}\right)-$ forces their energies to be almost equal when compared to their total energy $\sqrt{s} / 2$, thus forcing $x_{-} \simeq x_{+}$and suppressing the region of support of the $x_{-}$integral. However, this momentum constraint applies to the quarks in their own c.m. frame, but their relative momentum when evaluated in the $e^{+} e^{-}$c.m. frame must be multiplied by a relativistic boost factor $\gamma(v) v \approx 1 / 2 \cdot \sqrt{s / s_{1}}$. The whole [0,1] interval for the $x_{-}$integral therefore contributes to hadronic bound states.

One is therefore left to consider the $s_{1}$ integral. Strictly speaking, the allowed range of $s_{1}$ for a vector state $V^{0}$ of narrow width is vanishingly small, and $\left|G_{V}\right|^{2}$ assumes the form of a decay constant $F_{V^{0}}^{2}$ (of dimension mass squared) times a delta function $\delta\left(s_{1}-m_{V^{0}}^{2}\right)$ : Requiring the virtual photon in Fig. 3 to produce only a single exclusive state $V^{0}$ of squared mass $s_{1}$ fixes the photon virtuality precisely to equal $s_{1}$. However, the same result is obtained if $G_{V}\left(s_{1}\right)$ is replaced by a properly normalized Breit-Wigner distribution representing a wide state such as $\rho^{0}$. The form factor $\left|G_{V}\right|^{2}$ must also decrease with $s_{1}$ in order to satisfy unitarity, but this decrease merely indicates that couplings to highly excited $V^{0}$ 's must decrease with $s_{1}=m_{V^{0}}^{2}$ in order to sum to a finite total. In the AdS/QCD calculation of Ref. [7], this dependence in terms of the AdS/QCD scale parameter $\kappa$ would $\mathrm{read} \kappa^{2} / s_{1}$. The expected "large" momentum-scale suppression in $G_{V}$ in exclusive transitions due to constituent counting rules actually comes from $s_{1}$.

Knowledge of the larger scale $|t|$ by the $V^{0}$ is lost in the propagation of the photon. For the most naive form of the counting rules to hold, all propagators and fermion couplings must contribute large scales to the amplitude, and the virtual photon in this case contributes only $1 / s_{1}$. One concludes that the forward cross section for a strictly twobody process $e^{+} e^{-} \rightarrow \gamma V^{0}$ in which $V^{0}$ contains two fundamental constituents $[N=5$ in Eq. (1)] should scale as $d \sigma / d t \sim\left(\alpha^{3} / s|t|\right)\left|G_{V}\left(s_{1}\right)\right|^{2}$, with $G_{V}\left(s_{1}\right) \sim 1 / \sqrt{s_{1}}$.

In contrast, Ref. [7] concluded that $G_{V}\left(s_{1}\right) \sim 1 / \sqrt{|t|}$, the large scale $|t|$ reemerging through a hard-gluon exchange needed to bind the otherwise noncollinear pair $q \bar{q}$ into the bound state $V^{0}$. However, as noted above, the formation of a single photon of virtuality $s_{1} \ll|t|$ completely erases the system's memory of the large scale $|t|$ : Emission of a hard gluon in this case is not natural. Moreover, even though the $q \bar{q}$ pair can have $O(\sqrt{s})$ energies in the c.m., their momentum invariants (their masses and $s_{1}$ ) are small.

Consider instead a process such as that illustrated in Fig. 3, except that the photon virtuality $s_{1}$ does not precisely equal $m_{V^{0}}^{2}$ but rather assumes a value of $O(|t|)$ (because the process is still one of forward scattering). The inclusive process $e^{+} e^{-} \rightarrow \gamma q \bar{q}$ has a much greater phase space than does the exclusive process $e^{+} e^{-} \rightarrow \gamma V^{0}$, and its cross section scales in the forward direction as $d \sigma / d t \sim$ $1 / s|t|^{2}$ (as seen above for $e^{-} e^{+} \rightarrow \gamma \mu^{+} \mu^{-}$). This inclusive rate does indeed include a portion of the exclusive channel $e^{+} e^{-} \rightarrow \gamma V^{0}$, but only from the large- $|t|$ tail of the $V^{0}$ line shape. It also includes contributions from $e^{+} e^{-} \rightarrow \gamma V^{0}$ plus additional soft hadrons such that the total hadronic system has invariant mass squared of $O(|t|)$, which can be misidentified as the exclusive channel $e^{+} e^{-} \rightarrow \gamma V^{0}$ if the soft hadrons escape detection.

In summary, the correct high-momentum forward-angle scaling for the genuine two-body exclusive $e^{+} e^{-} \rightarrow \gamma V^{0}$ cross section is $d \sigma / d t \sim 1 / s|t|$, rather than $1 / s|t|^{2}$ as given in Ref. [7]. However, tails of the original process and processes that can be misidentified as $e^{+} e^{-} \rightarrow \gamma V^{0}$ give contributions scaling as $1 / s|t|^{2}$; and since they have much greater available phase space, they may dominate the observed rate of $e^{+} e^{-} \rightarrow \gamma V^{0}$ even if $|t|$ is rather larger than $m_{V^{0}}^{2}$. Completely analogous comments hold for the process $e^{+} e^{-} \rightarrow V_{a}^{0} V_{b}^{0}$.

\section{INCLUSIVE $e^{+} e^{-}$PROCESSES WITH VIRTUAL PHOTONS VS. VMD}

When the $V^{0}$ s are off shell, an extra power falloff in the large scale appears for each meson state. The forwardscattered virtual photons then carry $O(|t|)$ momentum transfers, and the constituent counting rules read [7]

$$
\begin{aligned}
\frac{d \sigma}{d t}\left(e^{+} e^{-} \rightarrow \gamma V^{* 0}\right) & \sim \frac{1}{s^{2}|t|^{2}}, \\
\frac{d \sigma}{d t}\left(e^{+} e^{-} \rightarrow V_{a}^{* 0} V_{b}^{* 0}\right) & \sim \frac{1}{s^{2}|t|^{3}} .
\end{aligned}
$$

Crucially, the full QCD theory differs from an effective field theory developed from VMD in their applications to physical processes. In particular, VMD makes no distinction between on-shell and off-shell $V^{0}$, which leads to an incorrect off-shell behavior of the $\gamma^{*} \rightarrow V^{0}$ transition. In Ref. [7] we explicitly showed that a constant value for this transition is ruled out by the nontrivial form factor $G_{V}\left(q^{2}\right)$, where $q$ is the photon (or $V^{0}$ ) four-momentum. In fact, $G_{V} \sim 1 /|t|^{1 / 2}$ at large $t=q^{2}$, consistent with perturbative $\mathrm{QCD}(\mathrm{pQCD})$ and constituent counting rules. 


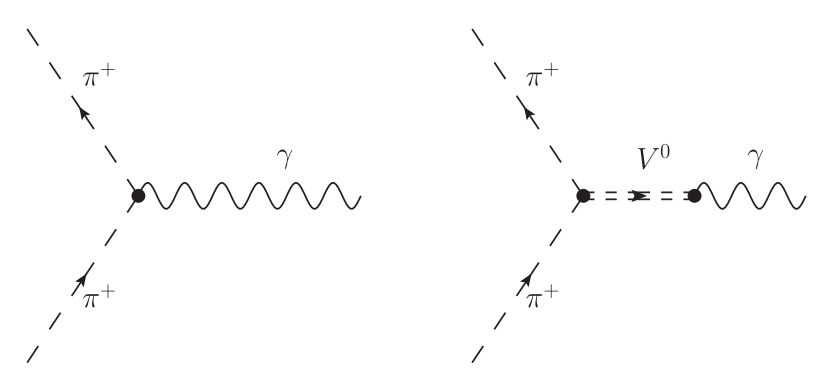

FIG. 4. Diagrams (contact and vector-meson exchange) contributing to the electromagnetic form factor of the pion in effective field theories involving vector mesons as elementary fields.

In Ref. [7] we pointed out another property distinguishing pQCD from effective field theories treating $V^{0}$ as elementary fields, i.e., in its application to electromagnetic form factors of hadrons. Let us review this important point: In effective theories (like VMD [11], chiral perturbation theory [18], or the hidden-symmetry approach with vector mesons as dynamical gauge bosons [19]) that treat the $V^{0}$ as an elementary field, the form factor $G_{V}\left(q^{2}\right)$ is a constant. One obtains different scaling contributions of the relevant diagrams with elementary $V^{0}$ and with pQCD. For example, in the case of the pion electromagnetic form factor $F_{\pi}\left(Q^{2}\right)$, one may split the VMD result into the contact and the vector-meson exchange diagrams (Fig. 4). The contact diagram gives 1 (a constant contribution at large $Q^{2}=-q^{2}$ ), whereas the vector-meson exchange diagram gives a $(-1+$ $\left.m_{V^{0}}^{2} / Q^{2}\right)$ contribution. Summing these together, one arrives at an $m_{V^{0}}^{2} / Q^{2}$ scaling. In contrast, using pQCD counting rules, the contact diagram turns out to be of leading order $\left(1 / Q^{2}\right)$, whereas the $V^{0}$-exchange diagram is subleading $\left[\left(1 / Q^{2}\right)^{3 / 2}\right]$ at large $Q^{2}$. Of course, at fixed $Q^{2}=m_{V^{0}}^{2}$ the contributions do not cleanly separate. Another crucial point is that, while the scaling of $F_{\pi}\left(Q^{2}\right)$ in VMD is formally $m_{V^{0}}^{2} / Q^{2}$ due to the $V^{0}$ propagator, this factor has no connection with $1 / Q^{2}$ scaling in $\mathrm{pQCD}$ due to hard-gluon exchanges between constituent quarks in the pion. Therefore, the $1 / Q^{2}$ falloff of $F_{\pi}\left(Q^{2}\right)$ in VMD coincides with pQCD accidentally and is not related to the physical nature of strong interactions at high scales. As a consequence, an effective field theory of VMD completely fails in the description of the electromagnetic form factors of baryons and multiconstituent hadronic systems (tetraquarks, pentaquarks, etc.) at high scales. Without $\mathrm{pQCD}$ it is impossible to produce the $1 / Q^{2(N-1)}$ falloff of the electromagnetic form factors of hadrons containing $N$ constituents. Note that a criticism of VMD in the description of data for photon-hadron interactions at high energies was also stressed in Ref. [20]. In particular, Ref. [20] argues that VMD is not a suitable framework for a description of deep-inelastic scattering over the full kinematical range.

Additionally, in the generic case of exclusive on-shell hadron production, it is not possible to approximate the hadrons as elementary fields. In the recent paper Ref. [21], the exclusive production processes of scalar $S=0^{++}$and tensor $T=2^{++}$mesons through single-photon annihilation $e^{+} e^{-} \rightarrow \gamma^{*} \rightarrow \gamma S(T)$ were analyzed. Here, the transition form factors of $\gamma^{*} \rightarrow \gamma S$ and $\gamma^{*} \rightarrow \gamma T$ are not constants, and they scale as $F_{\gamma^{*} \gamma S}(s) \sim 1 / s$ and $F_{\gamma^{*} \gamma T}(s) \sim 1 / s^{2}$ at large $s$, consistent with the scaling of the corresponding form factors at large values of virtual-photon squared Euclidean momentum $Q^{2}: F_{\gamma^{*} \gamma S}\left(Q^{2}\right) \sim 1 / Q^{2}$ [22] and $F_{\gamma^{*} \gamma T}\left(Q^{2}\right) \sim$ $1 / Q^{4}$ [23]. The scaling of the form factors follows directly from using the differing twist counting for the $S$ - and $T$ creating operators [12]. As a result, both differential cross sections scale as $\frac{d \sigma}{d t}\left(e^{+} e^{-} \rightarrow \gamma+S(T)\right) \sim 1 / s^{3}$, in agreement with constituent-quark counting rules that treat real scalar and tensor mesons as $q \bar{q}$ systems with $N_{S(T)}=2$ substituted into the counting formula (1) for $d \sigma / d t$. Consistent with Ref. [6], when scalar and tensor mesons are considered as tetraquark systems of two tightly bound color diquarks, the corresponding transition form factors and differential cross sections have the same falloffs as in the $q \bar{q}$ case. For other tetraquark or two-hadron molecular configurations, the transition form factors $F_{\gamma^{*} \gamma S(T)}(s)$ and the differential cross section $d \sigma / d t$ have additional falloffs scaling as $1 / s$ and $1 / s^{2}$, respectively.

Again, we point out that the case of single and double neutral vector-meson production via an intermediate photon or the weak gauge boson fields is very specific, constraining $V^{0}$ (with respect to the counting rules) to acting as effectively fundamental (structureless) particles; it is the result of an exceptional case in which some of the internal propagators (i.e., virtual gauge bosons) are explicitly excluded from carrying large off-shell virtuality.

\section{VECTOR-MESON PRODUCTION IN $p p$ SCATTERING}

In this section we discuss $V^{0}$ production in $p p$ scattering processes. The hadronic angular momentum dependence of hard exclusive QCD processes is controlled by the Brodsky-Lepage helicity selection rules [12,24], which state that the total hadron helicity is conserved from the initial to the final state, up to higher-twist corrections appearing as inverse powers of the hard scale. This result was used for $e^{+} e^{-} \rightarrow V^{0} P^{0}$ in Sec. II. Taking here $V^{0}=\rho^{0}$, we consider three specific cases of semi-inclusive $\rho^{0}$-meson production $[4,25,26]$ : (1) the reaction $p p \rightarrow \rho^{0} X$, with the $\rho^{0}$ produced from jet fragmentation and $X$ being any hadrons; (2) the reaction $p p \rightarrow \rho_{D}^{0} X$, with a "direct" $\rho_{D}^{0}$ produced at high $p_{T}$ in isolation from other hadrons on the trigger side (i.e., without any same-side particles); and (3) the reaction $p p \rightarrow \gamma^{*} X \rightarrow \rho_{D}^{0} X$, where a single virtual photon produces a "direct" $\rho^{0}$, which again is isolated on the trigger side.

Reaction (1) has normal conformal scaling (modulo log corrections). Consistent with Eq. (1), the differential cross 
section for semi-inclusive production of a single hadron $\rho^{0}$ with form factor $F$ scales as

$$
\frac{d \sigma}{d^{3} p / E} \sim \frac{F\left(x_{T}\right)}{p_{T}^{4}}
$$

where $p_{T}$ and $x_{T}=2 p_{T} / \sqrt{s}$ are the transverse momentum and its light-cone fraction, respectively.

In case (2) the $\rho_{D}^{0}$ couples via a $q \bar{q}$ to the hard underlying hadron subprocess. The corresponding differential cross section then has an additional $1 / p_{T}^{2}$ falloff in comparison with case (1) and scales as

$$
\frac{d \sigma}{d^{3} p / E} \sim \frac{F\left(x_{T}\right)}{p_{T}^{6}},
$$

reflecting the corresponding twist-2 operator and the $|q \bar{q}\rangle$ Fock state of the $\rho^{0}$. Note that reaction (2) is power suppressed at high $p_{T}$ (being higher twist), but the $\rho^{0}$ in this case exhibits color transparency [27]: It is produced directly from the hard subprocess as a small-sized colorsinglet state and can propagate through a nuclear medium with minimal interactions. In contrast to reaction (2), the process $p p \rightarrow \gamma X$ with an isolated photon occurs at leading twist since the photon can couple directly to the hard process without additional power suppression.

Consideration of $p p \rightarrow \gamma X$ leads to case (3), which again scales like $\frac{d \sigma}{d^{3} p / E} \sim 1 / p_{T}^{4}$ as in Eq. (6), since in this case the $\rho^{0}$ couples softly via the twist- 1 photon field to the hard subprocess without an additional power suppression. Reaction (3) exhibits the same type of behavior as discussed in Secs. II and III for $e^{+} e^{-} \rightarrow \gamma V^{0}$ and $e^{+} e^{-} \rightarrow V_{a}^{0} V_{b}^{0}$.

\section{CONCLUSIONS}

Let us summarize the main results of this paper. We examined the application of QCD constituent counting rules to exclusive processes involving neutral vector mesons $V^{0}$. In particular, we considered exclusive production of one or two $V^{0}$ via intermediate photons from $e^{+} e^{-}$ annihilation and in $p p$ scattering. In the case of a real $V^{0}$, the photon virtuality $s_{1}$ can be fixed to a precise value $m_{V^{0}}^{2}$, in effect treating the $V^{0}$ as a single fundamental particle.
Therefore, each real $V^{0}$ contributes to the constituent counting rules with $N_{V_{0}}=1$. Because the leading operator underlying the $V^{0}$ has twist 1 , in the case of single or double on-shell $V^{0}$ production via intermediate photons, the predicted scaling from counting rules coincides with $\mathrm{VMD}$, an effective theory that treats vector mesons as elementary fields.

However, the VMD prediction fails in the general case where the $V^{0}$ is not coupled solely through an elementary photon field, and in that case, the leading-twist interpolating operator has $N_{V_{0}}=2$. Furthermore, VMD fails in the case of off-shell coupling of the electromagnetic field with hadrons at large momentum scales because this approach, by construction, does not respect the constituent structure of hadrons and hard-gluon exchange at large scales. As a result, the large- $Q^{2}$ scaling of electromagnetic form factors of hadrons with $N \geq 3$ constituents in VMD is not consistent with that from pQCD. Only in the case of $q \bar{q}$ systems (conventional mesons) is the VMD prediction of $1 / Q^{2}$ scaling formally similar to that of $\mathrm{pQCD}$ because of the $1 / Q^{2}$ behavior of the $V^{0}$ propagator. One should also note the criticism of VMD in the description of data for photon-hadron interactions at high energies stressed before in Ref. [20].

\section{ACKNOWLEDGMENTS}

We thank Feng-Kun Guo for suggesting the examination of the process $e^{+} e^{-} \rightarrow \gamma \mu^{+} \mu^{-}$. This research was supported by the U.S. Department of Energy, Contract No. DE-AC02-76SF00515 (S. J. B.), by the U.S. National Science Foundation under Grant No. PHY1403891 (R.F. L.), by the German Bundesministerium für Bildung und Forschung (BMBF) under Project 05P2015-ALICE at High Rate (BMBF-FSP 202): "Jet and fragmentation processes at ALICE and the parton structure of nuclei and structure of heavy hadrons," by CONICYT (Chile) PIA/Basal FB0821, by Tomsk State University Competitiveness Improvement Program, by the Russian Federation program "Nauka" (Contract No. 0.1764.GZB.2017), and by Tomsk Polytechnic University Competitiveness Enhancement Program (Grant No. VIU-FTI-72/2017) (V.E. L.). SLACPUB-17184.
[1] S. J. Brodsky and G. R. Farrar, Phys. Rev. Lett. 31, 1153 (1973).

[2] V. A. Matveev, R. M. Muradian, and A. N. Tavkhelidze, Lett. Nuovo Cimento Soc. Ital. Fis. 7, 719 (1973).
[3] G. P. Lepage and S. J. Brodsky, Phys. Rev. D 22, 2157 (1980).

[4] D. W. Sivers, S. J. Brodsky, and R. Blankenbecler, Phys. Rep. 23, 1 (1976). 
[5] S. J. Brodsky, G. F. de Teramond, H. G. Dosch, and J. Erlich, Phys. Rep. 584, 1 (2015).

[6] S. J. Brodsky and R. F. Lebed, Phys. Rev. D 91, 114025 (2015).

[7] S. J. Brodsky, R. F. Lebed, and V. E. Lyubovitskij, Phys. Lett. B 764, 174 (2017).

[8] H. Kawamura, S. Kumano, and T. Sekihara, Phys. Rev. D 88, 034010 (2013).

[9] W.-C. Chang, S. Kumano, and T. Sekihara, Phys. Rev. D 93, 034006 (2016).

[10] F.-K. Guo, U.-G. Meißner, and W. Wang, Chin. Phys. C 41, 053108 (2017).

[11] J. J. Sakurai, Currents and Mesons (University of Chicago Press, Chicago, 1969).

[12] S. J. Brodsky and G. P. Lepage, Phys. Rev. D 24, 2848 (1981).

[13] F. A. Berends and R. Kleiss, Nucl. Phys. B177, 237 (1981).

[14] E. A. Kuraev and G. V. Meledin, Nucl. Phys. B122, 485 (1977).

[15] A. B. Arbuzov, E. Bartos, V. V. Bytev, E. A. Kuraev, and Z. K. Silagadze, Pis'ma Zh. Eksp. Teor. Fiz. 80, 806 (2004) [JETP Lett. 80, 678 (2004)].
[16] S. J. Brodsky and R. F. Lebed, Phys. Rev. Lett. 102, 213401 (2009).

[17] M. E. Peskin and D. V. Schroeder, An Introduction to Quantum Field Theory (Westview Press, New York, 1995).

[18] G. Ecker, J. Gasser, H. Leutwyler, A. Pich, and E. de Rafael, Phys. Lett. B 223, 425 (1989).

[19] M. Bando, T. Kugo, and K. Yamawaki, Phys. Rep. 164, 217 (1988).

[20] J. I. Friedman, Rev. Mod. Phys. 63, 615 (1991).

[21] A. G. Chumakov, T. Gutsche, V.E. Lyubovitskij, and I. Schmidt, Phys. Rev. D 96, 094018 (2017).

[22] P. Kroll, Eur. Phys. J. C 77, 95 (2017).

[23] N. N. Achasov, A. V. Kiselev, and G. N. Shestakov, Pis'ma Zh. Eksp. Teor. Fiz. 102, 655 (2015) [JETP Lett. 102, 571 (2015)].

[24] S. J. Brodsky and G. P. Lepage, Adv. Ser. Dir. High Energy Phys. 5, 93 (1989).

[25] S. J. Brodsky, M. Diehl, P. Hoyer, and S. Peigne, Phys. Lett. B 449, 306 (1999).

[26] F. Arleo, S. J. Brodsky, D. S. Hwang, and A. M. Sickles, Phys. Rev. Lett. 105, 062002 (2010).

[27] S. J. Brodsky and A. Sickles, Phys. Lett. B 668, 111 (2008). 